\title{
Pobreza laboral y desempleo en España 2009-2019
}

JUAN HERRERA BALLESTEROS *

Iberoamerican Journal of Industrial Relations

ISSN 2173-6812

VOL. 38, (2020)

pp. 28-39

D. pundeses 


\section{FECHA RECEPCIÓN:}

2020-02-05

FECHA REVISIÓN:

2020-02-18

\section{FECHA ACEPTACIÓN:}

2020-10-04

FECHA PUBLICACIÓN:

2020-12-05

\section{ReSUMEN}

Uno de los objetivos recogidos en la Estrategia de Europa 2020 era reducir, en el período 2009-2019, 20 millones de personas de las registradas en riesgo de pobreza y de exclusión social. El compromiso de España fue rebajar en el mismo período, entre 1.400 .000 y 1.500 .000 personas. Asimismo, la pobreza tradicionalmente se ha relacionado con el desempleo o situaciones de inactividad y aunque sin duda siguen siendo factores de riesgo de pobreza, en la actualidad, tener un trabajo no es óbice para evitar caer en riesgo de pobreza o exclusión social. Este trabajo, a través de una revisión de la literatura, tiene como objetivo contextualizar la situación de la pobreza y exclusión social en España y profundizar en el análisis de la pobreza laboral. Las conclusiones sugieren, que España no sólo no ha cumplido su compromiso en cuanto a reducir personas en riesgo de pobreza y exclusión, sino que además está por encima del punto de partida y de la media europea. El desempleo tiene un fuerte impacto sobre la pobreza en cuanto a los hogares en los que todos sus miembros están desempleados. El número de trabajadores pobres es mayor que el de los desempleados pobres. Por último, os trabajadores por cuenta propia tienen un mayor riesgo de pobreza laboral que los trabajadores por cuenta ajena.

\section{Palabras Clave}

Pobreza laboral, desempleo, pobreza dinámica, pobreza y exclusión social

\section{Abstract}

One of the objectives set out in the Europe 2020 Strategy was to reduce, in the period 2009-2019, 20 million of those registered as being at risk of poverty and social exclusion. Spain's commitment was to reduce between $1,400,000$ and 1,500,000 people in the same period. Furthermore, poverty has traditionally been related to unemployment or situations of inactivity and although they undoubtedly continue to be risk factors for poverty, at present, having a job does not prevent one from falling into the risk of poverty or social exclusion. This paper, through a review of the literature, aims to contextualize the situation of poverty and social exclusion in Spain and to deepen the analysis of working poverty. The conclusions suggest that Spain has not only failed to meet its commitment to reduce people at risk of poverty and exclusion, but is also above the starting point and the European average. Unemployment has a strong impact on poverty in terms of households where all members are unemployed. The number of working poor is higher than the number of unemployed poor. Finally, the self-employed have a higher risk of in-work poverty than employed people.

\section{KeY WORDS}

Working poverty, unemployment, dynamic poverty, poverty and social exclusion
* juanherrera@uma.es

Dpto. de Economía y Administración de Empresas.

Universidad de Málaga, España.

ORCID 0000-0003-0564-9338

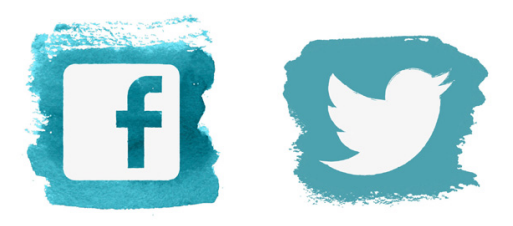




\section{POBREZA LABORAL Y DESEMPLEO EN ESPAÑA 2009-2019}

\section{Introducción}

La Organización Internacional del Trabajo (OIT) define el concepto de trabajador pobre dentro del concepto subempleo. Explica que pertenecerían a esa categoría aquellos trabajadores asalariados con un salario que no se corresponde con las expectativas y características de trabajador (Martínez-Martín, García-Moreno \& Lozano-Martín, 2018).

La situación de los trabajadores pobres en buena parte de los Estados miembros de Europa y, principalmente en España, se antoja estructural. El interés y la atención a la pobreza labora es reciente en Europa. La pobreza tradicionalmente se ha relacionado con el desempleo o situaciones de inactividad (Peñas-Casas y Latta, 2004; Strengmann-Kuhn, 2002) y, aunque sin duda siguen siendo factores de riesgo de pobreza, surgen nuevas formas de empobrecerse tales como el trabajo a tiempo parcial o la pobreza de bajos salarios (Ayala-Cañón et al, 2008), que hacen que la relación entre ocupación y pobreza no siga las mismas pautas de hace décadas y que el trabajo no sea siempre suficiente para evitar la pobreza (Ponthieux, 2010; Airio, 2008 Bardone y Guio, 2005; Tejero, 2018).

En los países del sur de Europa, los mercados laborales son fundamentalmente dualistas (Halleröd et al., 2015; Visser, 2009), se caracterizan por ser un mercado de trabajo rígido con diferentes niveles de protección, muchos derechos para los que están en el centro del mercado de trabajo (contratos indefinidos) y pocos para los de la periferia (contratos temporales) (Rodríguez-Cabrero, 2011), provocando una tasa alta de temporalidad, alta rotación laboral y como consecuencia, el aumento del empleo precario o de baja calidad, incidiendo en un mayo riesgo de pobreza laboral.

España, a pesar de haber vivido un ciclo de expansión económica y, como consecuencia crecimiento de empleo, no ha sido capaz de reducir la pobreza y/o exclusión social, ni la pobreza laboral. Tras ese ciclo de expansión, en 2007 irrumpe una crisis en la que un mercado laboral restrictivo, junto con iniciativas políticas controvertidas como, la Reforma Laboral de febrero de 2012 - el sistema de negociación colectiva se vio debilitado, en cuanto a la negociación de los salarios por parte de los representantes de los trabajadores_-, provocaron una bajada de sala- rios y mayor desigualdad salarial (Martínez-Martín, García-Moreno \& Lozano-Martín, 2018), que probablemente haya empeorado la situación de la pobreza laboral en España.

En el $9^{\circ}$ Informe de European Anti Poverty Network, de octubre de 2019, Llano (2019) pone de manifiesto que es muy significativo que haya más personas pobres que trabajan, es decir, que disponen de un empleo remunerado, pero cuyo salario no les permite disponer de recursos necesarios para satisfacer sus necesidades, que las que se encuentran en desempleo.

El presente trabajo, a través de una revisión de la literatura, tiene como objetivo contextualizar la situación de la pobreza y exclusión social en España, para luego profundizar en la pobreza laboral y el desempleo. La organización de este trabajo es la siguiente; el segundo apartado, está orientado a la contextualización de la pobreza y exclusión social en Europa y en España, tambien expone incidencias del desempleo en la pobreza, el tercer apartado, aporta una visión de la conceptualización del trabajador pobre y plantea la repercusión del mercado laboral y los recortes de los derechos laborales como factores que impulsan las probabilidades de riesgo de pobreza y exclusión social, el cuarto apartado, trata de explicar el concepto de pobreza dinámica y exponer diferentes visiones de la literatura sobre el desarrollo del mismo, así como de exponer determinados resultados de trabajos sobre la pobreza dinámica en España, en el quinto y último capítulo, nos encontramos con las principales conclusiones y el planteamiento de posibles propuestas.

\section{Pobreza y desigualdad. Contexto Europeo.}

En 2010 el Consejo Europeo adoptó oficialmente la Estrategia 2020 y estableció como objetivo principal que salieran de la pobreza y/o de la exclusión social, al menos, 20 millones de personas para 2020. Para medir la pobreza y/o exclusión social la UE propone el indicador AROPE (At Risk Of Poverty and/or Exclusion) que hace referencia al porcentaje de población que se encuentra en riesgo de pobreza y/o exclusión social. Se compone de tres medidas distintas, considerándose dentro de esta clasificación cuando se encuentran en al menos una de las situaciones siguientes; a) Personas en riesgo de pobreza, es decir, con una renta disponible por debajo del umbral de riesgo de pobreza; b) Personas que sufren Privación Material Severa (PMS), es decir, las que no pueden permitirse al menos cuatro de los nueve artículos materiales predefinidos ${ }^{1}$ que la mayoría de las personas

1. Los artículos predefinidos son: la capacidad de hacer frente a gastos inesperados; permitir una semana de vacaciones anuales fuera de casa; permitirse una comida con carne, pescado o un equivaente vegetariano cada dos días; calentar adecuadamente la su vivienda; comprar una serie de bienes duraderos como una lavadora, un televisor en color, un teléfono o un coche; pagar puntualmente una hipoteca, el alquiler; las facturas de los servicios públicos u otros pagos de préstamos (Eurostat, 2018).

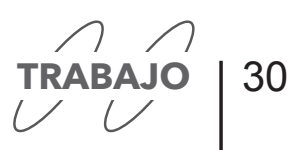


consideran deseables o incluso necesarios para llevar una calidad de vida adecuada y; c) Las personas de 0 a 59 años que viven en un hogar con una baja intensidad de trabajo (BITH), es decir, aquellos que viven en hogares donde los adultos no trabajaron más del $20 \%$ de su pleno potencial de trabajo durante el año anterior (Eurostat, 2018).

Entre los habitantes de la UE-28 que se enfrentan al riesgo de pobreza o exclusión social en 2016, 8,4 millones de personas vivían en hogares que experimentan simultáneamente los tres criterios de pobreza y exclusión social (Figura 1). Por otra parte, existe un importante número de hogares que simultanean al menos dos de los tres criterios, en este sentido, 15,1 millones de personas se encuentran en riesgo de pobreza y a la vez vive en un hogar con una intensidad de trabajo muy baja. Significativos son también los 11,5 millones que simultanean la carencia material severa con el riesgo de pobreza y, alrededor de 2,5 millones de personas vivían en hogares con una intensidad de trabajo muy baja y a la vez sufrían carencia material severa.

Por último, la mayoría de la población de la UE-28 que vive en riesgo de pobreza exclusión social experimentó sólo y exclusivamente uno de los tres criterios individuales Así, en 2016 se encontraban 51,9 millones de personas que estaban exclusivamente en riesgo de pobreza, 15,5 millones que sufrían carencia material severa y 13,2 millones que vivían en hogares con una intensidad de trabajo muy baja (Eurostat, 2018).

Figura 1 Personas en riesgo de pobreza o exclusión social por tipo de riesgo EU-28 año 2016

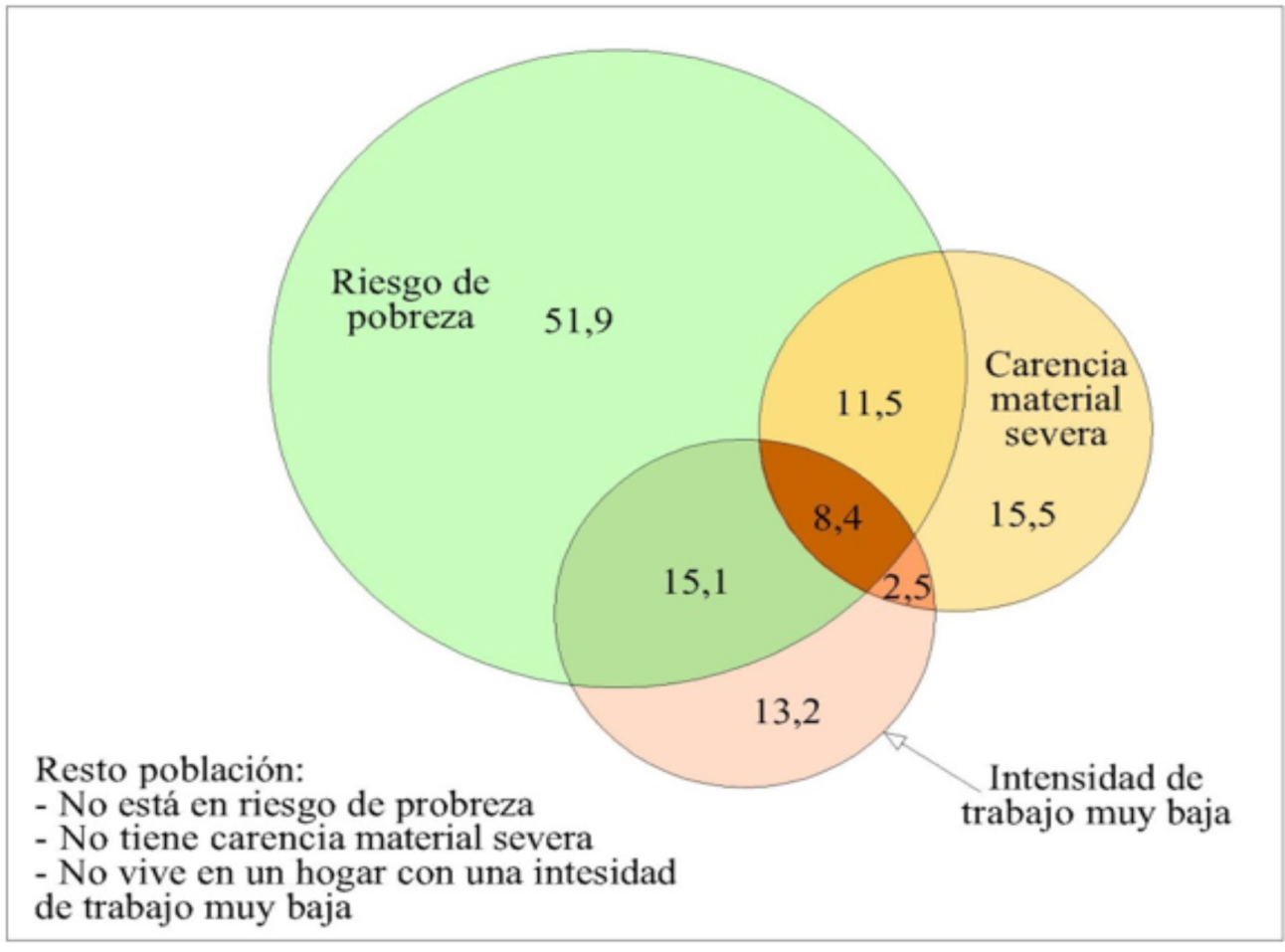

Fuente: Elaboración propia a partir de datos de Eurostat (2018)
Según las estimaciones de Eurostat (2018), en 2012 se contaba con un total de 123,6 millones de personas en riesgo de pobreza o exclusión social. En cuatro años se ha ido reduciendo de forma gradual bajando a los 118 millones de personas en 2016, es decir, el 23,5 $\%$ de la población de la UE-28, están en riesgo de pobreza y/o se enfrentan a graves privaciones de materiales y/o viven en un hogar con una intensidad laboral muy baja. En cuanto a la diferenciación de género, se confirma que el riesgo de pobreza o exclusión social es mayor entre las mujeres que entre los hombres. Con respecto a la edad, los jóvenes sufren mayor riesgo que las personas de mediana edad o los pensionistas. Por último, en cuanto al nivel de educación, aquellos con un menor nivel tienen más probabilidades de caer en la pobreza o exclusión social que los que tienen un nivel superior. Por otra parte, cuando se trata de las cargas familiares y del tipo de familia, los hogares unipersonales con hijos a cargo y las familias nucleares con más de dos hijos, en 2016 tenían más probabilidades de caer en la pobreza o la exclusión social. Bulgaria y Eslovenia son los Estados miembros de la UE-28 que representan mayor porcentaje de familias monoparentales en riesgo de pobreza o exclusión social y, Rumanía, Bulgaria y España, son los países donde se registra el mayor riesgo de pobreza y exclusión social, cuando se trata de hogares con niños. En la actualidad, tener un trabajo no es óbice para evitar caer en riesgo de pobreza o exclusión social, así en 2016 el 12,4\% de las personas de la UE-28 que tenían empleo estaban considerados dentro del colectivo con riesgo de pobreza o exclusión social. En el caso de las personas desempleadas se enfrentan a un riesgo especialmente alto, con un $67 \%$ de los desempleados que están en riesgo de pobreza o exclusión social (Gráfico 1).

Gráfico 1. Personas en riesgo de pobreza o exclusión social por características socioeconómica EU-28 año 2016

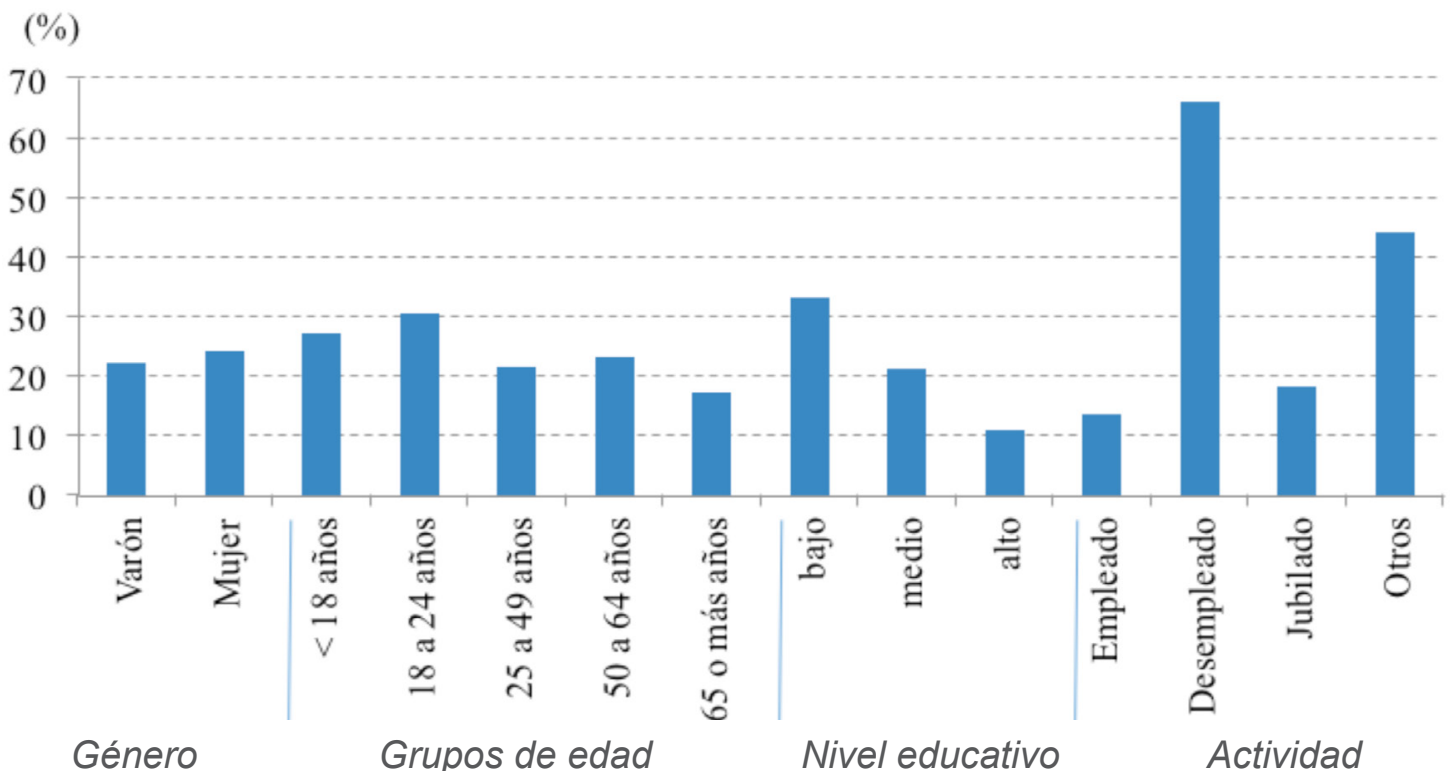

Fuente: Elaboración propia a partir de datos de Eurostat (2018) 
En la UE existe heterogeneidad en el riesgo de pobreza o exclusión social, tanto internamente en cada estado, como entre los propios miembros. Así, en los siguientes Estados miembros de Europa del Este o del Sur; Bulgaria, Rumanía, Grecia, Lituania, Letonia y Polonia, el riesgo de pobreza en las zonas rurales es mayor que en las urbanas, sin embargo, en países de Europa occidental tales como Bélgica, Austria, Dinamarca, Alemania y los Países Bajos, el riesgo de pobreza se concentra más en las ciudades. Para el caso de España, el 33,5\% de la población rural está en riesgo de pobreza y exclusión social frente al $25,9 \%$ de la población de las ciudades.

\section{a. Pobreza monetaria}

La tasa de riesgo de pobreza proporciona información de la dimensión monetaria de la pobreza y la exclusión social, trata de aportar la proporción de personas que tienen un nive de ingresos inferior al umbral nacional de riesgo de pobreza, que para la UE en general, el umbral establecido es el $60 \%$ de la renta nacional media equivalente ${ }^{2}$ (Eurostat, 2018).

En 2016, los Estados miembros con índices más altos en probabilidad de riesgo de pobreza y exclusión social eran Rumania, Bulgaria, España, Lituania, Letonia, Estonia y Grecia, todos oscilaban entre el $25,3 \%$ y el $21,3 \%$ de su población. Por el contrario, los países en los que su población estaba expuesta a menores probabilidades de riesgo fueron Países Bajos, Eslovaquia, Dinamarca, Finlandia, Chequia, Noruega e Islandia, cuyos porcentajes de la población oscilaban entre el $12,7 \%$ y el $9,6 \%$. Un factor de impacto en la pobreza monetaria son las transferencias sociales, estas son utilizadas como medidas de protección social y tratan de reducir la tasa de riesgo de la pobreza. En 2016 las transferencias sociales redujeron la tasa de riesgo en la población de la UE-28 de un 25,9\% a un $17,3 \%$, lo que significa una reducción de un $8,6 \%$. Esta medida permite evaluar los sistemas de bienestar de los diferentes países analizando la eficacia de las medidas de protección social. Los países donde las transferencias sociales tuvieron un impacto particularmente alto en 2016 fueron Irlanda (datos 2015), Austria, Reino Unido y Noruega donde la tasa de riesgo se redujo por encima del $12 \%$, en el otro lado de la balanza se encuentra Bulgaria, Rumanía, Grecia, la ex República Yugoslava de Macedonia (datos 2015) y en Turquía (datos 2015), con un impacto en torno al 5\% (Eurostat, 2018).

\section{b. Privación de material}

Otra de las dimensiones del indicador AROPE es la privación material. Este indicador analiza la incapacidad de permitirse un conjunto de artículos materiales predefinidos, que la mayoría de las personas consideran como deseables o incluso necesarios para llevar una calidad de vida adecuada. Esta perspectiva complementa los análisis de la pobreza monetaria arrojando luz sobre las carencias en los hogares que pueden llevar hacia la pobreza absoluta. En 2016 la tasa de privación material severa ${ }^{3}(7,5 \%)$ de los Estados miembros de la UE-28 fue generalmente más baja que las tasas de riesgo de pobreza $(17,3 \%)$. Los países con tasas más altas de privación de material severa fueron Bulgaria $(31,9 \%)$, Rumanía $(23,8 \%)$ y Grecia $(22,4 \%)$ y los que tuvieron una tasa menor fueron Luxemburgo $(2 \%)$ y Suecia $(0,8 \%)$. Profundizando en el análisis, entre las personas en situación de riesgo de la pobreza en la UE-28 en 2016, alrededor del 21,3\% tampoco podían permitirse una comida decente cada dos días y, una vez más, los hogares monoparentales son los más afectados y vulnerables, así el 15,7\% de la población de la UE-28 que vivía en hogares monoparentales sufría graves privaciones de materiales, por otra parte, casi dos tercios $(65,2 \%)$ de los que vivían en hogares unipersonales con hijos dependientes no pudieron hacer frente a gastos financieros inesperados.

\section{c. Riesgo persistente de pobreza}

La tasa de riesgo de pobreza persistente es un indicador que capta a los miembros de la sociedad que son particularmente vulnerables al riesgo persistente de pobreza durante períodos de tiempo realmente largos. Esta tasa, muestra la proporción de personas que se encuentran por debajo del umbral de pobreza y que también lo han estado durante al menos en dos de los tres años anteriores. Esencialmente trata de dar visibilidad a los hogares que tienen menos posibilidades de recuperarse debido a la permanencia reiterada por debajo del umbral del riesgo de pobreza. Esta situación es parecida al desempleo de larga duración y que está estrechamente relacionada con un mayor nivel de exclusión social. La tasa de riesgo de pobreza persistente en general, en 2016 era más frecuente entre la población que vivía en hogares monoparentales y unipersonales, y se situaba entre el $17,4 \%$ y el $21,9 \%$ de la población, sin embargo, en los hogares monoparentales de Malta y Luxemburgo, se dieron porcentajes mucho más altos, registrándose un $42,3 \%$ y un $51,5 \%$ respectivamente (datos de 2015), algo menos, en torno un tercio de la población en Bélgica (datos de 2015), Grecia (datos de 2015) e Irlanda (datos de 2015). En cuanto a los hogares unipersonales, las tasas más altas de riesgo se dieron en Estonia (42,9\%) y Bulgaria (34,3\%). Las tasas más bajas se dieron en las personas que formaban parte de hogares con dos o más adultos sin hijos a su cargo $(6,6 \%)$ seguido de los hogares con dos o más adultos con hijos dependientes (Eurostat, 2018).
2. Este umbral no mide la riqueza o la pobreza en sí mismas, sino que proporcionan información sobre los niveles de ingresos por debajo de los cuales se considera que la población tiene bajos ingresos, lo que no implica necesariamente un nivel o calidad de vida muy bajos Eurostat (2018)
3. La tasa de privación de material severa se define como la proporción de la población que no puede permitirse cuatro o más artículos de entre los 9 artículos que se considera para la tasa de privación de material (Eurostat, 2018) 


\section{d. Pobreza y exclusión social en España}

Adentrándonos en el contexto español, el objetivo acordado por España en el marco de la Estrategia Europa 2020 fue, de acuerdo al indicador AROPE, reducir en el período 2009-2019 entre 1.400 .000 y 1.500 .000 personas de las que están en riesgo de pobreza y exclusión social. Llano (2019) señala que, en 2019, a falta de un año de cumplirse el plazo, y a pesar de la mejora en los últimos cuatro años, no solo no ha cumplido el compromiso, sino que habría que reducir en dos puntos la tasa para llegar a la situación de partida. A estas alturas, en términos absolutos, habría que reducir el AROPE en algo más de 2,6 millones de personas en un año. En 2018, a pesar de mantenerse la tendencia negativa por cuarto año consecutivo, el 26,1\% del total de la población española (12.188.288 personas) se encuentran en riesgo de pobreza y/o exclusión social. En relación a Europa, España en 2017 y en la variación acumulada desde el 2018, prácticamente en todas las variables de pobreza y exclusión social, los datos españoles están por encima de la media del conjunto de la UE. La tasa AROPE española es el 4,2\% más alta que la media de la UE y se posiciona como la séptima más alta de los países miembros. En cuanto a los perfiles más vulnerables, con respecto al género, la tendencia desde 2014 de descenso por parte de los hombres ha provocado que para 2018 las mujeres estén dos puntos porcentuales por encima de los hombres ( $27 \%$ frente al $25 \%$ ) y, en cuanto a la edad, la tasa más elevada es la de los jóvenes de entre 16 y 29 años (33,8\%). Por otra parte, con respecto a la situación de la población extranjera, en 2016, el 60,1\% de extranjeros procedentes de fuera de la UE y el 47,3\% procedentes de la UE, estaban en situación de pobreza y/o exclusión social. Con respecto a cómo ha evolucionado AROPE en los hogares, son los hogares monoparentales los que sufren una mayor tasa de pobreza y/o exclusión social con el $50 \%$ y con un crecimiento en el último año de dos puntos porcentuales. Por último, hay que señalar que el perfil de las personas pobres es muy distinto al de la miseria, se trata de personas españolas (80,5\%), adultas $(77,6 \%)$, con un nivel educativo medio o alto (22,9\% con bachiller y el $13,4 \%$ con educación superior) y con trabajo. Es muy significativo que haya más personas pobres que trabajan $(32,6 \%)$ que las que se encuentran en desempleo $(26,4 \%)$. Concluyendo, "el desempleo no define exclusivamente la pobreza; por el contrario, el grupo más numeroso es el de las personas ocupadas, es decir, que disponen de un empleo remunerado, pero cuyo salario no les permite disponer de los recursos necesarios para satisfacer sus necesidades" (Llano, 2019).

\section{e. Incidencia desempleo en la pobreza}

Para comparar el riesgo de pobreza entre los miembros de la UE se identifican tres grupos de hogares pobres; los hogares pobres que trabajan, los hogares en los que todo sus miembros están desempleados, pero reciben alguna transferencia de la Seguridad Social, y los hogares que no obtienen ningún ingreso por trabajo y no reciben transfe- rencias sociales. Ayala, Cantó \& Rodríguez, (2017) sugieren que es más probable que la percepción individual del riesgo de pobreza esté más condicionada por la falta de ingresos que por la exclusión de los miembros del hogar del mercado laboral. Parece repetirse el mismo patrón con la tasa de pobreza severa, influenciada más por la proporción de hogares que no obtienen ingresos por trabajo ni perciben transferencias sociales.

La crisis económica mundial que irrumpió en 2007 tuvo un fuerte efecto sobre la pobreza severa. La relevancia de su impacto se observa cuando se explica la pobreza a través de la proporción de hogares en los que todos los miembros activos están desempleados o la distribución del desempleo dentro del hogar está concentrada principalmente entre los cónyuges y los demás miembros de la familia. Tras la crisis, el desempleo en España creció del 8,6 \% al 26,0 \% en sólo cinco años, y la proporción de hogares en los que todos los miembros activos estaban desempleados aumentó de 2,6 \% a 10,5\%. Los países desarrollados han elevado su preocupación sobre la severidad del efecto del desempleo en los hogares, excluyendo completamente a sus miembros del mercado laboral. La OCDE (2001) señala que las tasas de pobreza, en todos los países, están más relacionadas con las tasas de hogares sin trabajo que con las tasas de desempleo basadas en el individuo. No menos relevante es la apreciación que hace Gregg et al. (2010) cuando plantea la importancia que tiene el desempleo en los hogares debido a la transmisión de los efectos intergeneracionales de la pobreza, teniendo en cuenta, que los ingresos de los padres tienen efectos significativos en el bienestar futuro de los hijos. (Ayala, Cantó \& Rodríguez, 2017)

\section{Trabajadores pobres}

La Organización Internacional del Trabajo (OIT) define el concepto de trabajador pobre dentro de del concepto subempleo. El subempleo puede ser considerado desde dos puntos de vista: subempleo por insuficiencia de horas y subempleo por situaciones de empleo inadecuado (Mata, 1999). La OIT explica que pertenecerían a esa categoría aquellos trabajadores asalariados que tienen un nivel de renta, en términos de salario, que poco o nada se corresponde con las expectativas y características del trabajador. Salarios que pueden provocar que el trabajador en situación de subempleo trate de buscar empleos complementarios o abandonar el empleo que lo ha llevado a esa situación (MartínezMartín, García-Moreno \& Lozano-Martín, 2018).

El concepto de trabajadores pobres es complejo y plantea dos dimensiones: la individual —ligada a las condiciones de trabajo— y la de los hogares — relacionada con los recursos provenientes de la familia o con las condiciones del hogar al que pertenece- (Tejero, 2017; Martínez-Martín, García-Moreno \& Lozano-Martín, 2018). Aragón et al (2012) añade la perspectiva de los niveles de renta (rentas salariales y otros recursos) 
En cuanto a los trabajadores pobres, no se hace distinción alguna entre los que trabajan por cuenta ajena o por cuenta propia. Según la definición ofrecida por Eurostat, se trata de personas ocupadas durante al menos siete meses con un mínimo de quince horas a la semana, y que pertenecen a hogares cuya renta anual disponible se encuentra por debajo del umbral de la pobreza.

En cuanto al peso de las dimensiones individual y el hogar, la literatura sugiere que las circunstancias de la segunda son más relevantes e inciden más en la pobreza que la propia dimensión individual. La estructura familiar cuenta con mayor capacidad explicativa y mayor influencia que las características del propio empleo para determinar la pobreza laboral (García e Ibáñez, 2007). Esta afirmación entronca con la idea de que no todos los trabajadores que tienen un salario bajo son pobres, debido a que va a depender de la situación que vivan en sus hogares, en relación al número de miembros y de los salarios y ayudas públicas que estos perciban. La intensidad laboral que tenga la unidad familia junto con posibles cambios que se puedan producir en las rentas que provienen del trabajo son los factores que, en mayor medida, podrían incidir en la aparición y/o persistencia de situaciones de pobreza laboral (Martínez-Martín, García-Moreno \& Lozano-Martín 2018). La intensidad laboral en los hogares se convierte en un factor explicativo de posiciones sociales tendentes a situaciones de pobreza. Existe una mayor probabilidad de pobreza en aquellos hogares donde exista una menor o más baja participación laboral de sus miembros. En España, el impacto del desempleo junto con el aumento de la presencia de situaciones de paro entre miembros del hogar ha tenido su impacto en la renta media y ésta sobre el aumento de la pobreza laboral (Tejero, 2017).

García e Ibánez (2007) trata de perfilar tres tipos trabajador pobre relacionando el salario percibido con la dependencia del hogar y viceversa. En primer lugar, aquel trabajado que vive en un hogar donde los ingresos son inferiores al umbral de pobreza y que reciben una remuneración baja por hora trabajada. En segundo lugar, sería aquel que teniendo un salario/hora bajo, cuenta con una estructura familiar que lo protege ante situaciones de riesgo. En tercer lugar, se refiere a los trabajadores pobres que no teniendo un salario/ hora bajo, sí conviven en un hogar que se calificaría pobre como consecuencia de situaciones familiares de exclusión. La participación laboral de los miembros adultos del hogar con respecto a la probabilidad de caer o de evitar dicha situación, es fundamental.

Por último, hay que señalar que el interés o la atención a la pobreza laboral en Europa es reciente. La existencia de los regímenes de bienestar y la intención de proteger los trabajos de mayor calidad hacían pensar que la pobreza estaba determinada por las situaciones de inactividad o desempleo (Peñas-Casas y Latta, 2004; Strengmann-Kuhn, 2002). Este patrón se sigue manteniendo, pero surgen nuevas formas de empobrecerse (trabajo a tiempo parcial, inactividad y pobreza de bajos salarios) (Ayala-Cañón et al, 2008) que hacen que la relación entre ocupación y pobreza no siga las mismas pautas de hace dé- cadas y que el trabajo no sea siempre suficiente para evitar la pobreza (Ponthieux, 2010; Airio, 2008; Bardone y Guio, 2005; Tejero, 2018)

\section{a. Repercusión del mercado laboral y los recortes de los derechos laborales}

El sistema de negociación colectiva del país, así como del modelo específico de Estado de Bienestar van a ser determinantes sobre la evolución y la incidencia de la desigualdad salarial y sobre el trabajador pobre (Hans-Jürgen y Lohmann, 2008; García y Arranz, 2014).

La OIT (2015) señala que en aquellos países donde la negociación colectiva está más centralizada y los trabajadores están bajo convenios colectivos o del sector, la desigualdad salarial es menor o tiene menor incidencia. En el caso de España, como consecuencia de la Reforma Laboral de febrero de 2012 (Real Decreto 3/2012 de 10 de febrero) se ha establecido la posibilidad de dar prioridad al convenio colectivo de empresa frente al convenio colectivo supra - empresarial, lo que ha permitido una mayor desigualdad salarial, consagrando la descentralización en el ámbito de la negociación colectiva (MartínezMartín, García-Moreno \& Lozano-Martín, 2018)

En cuanto a los mercados laborales, los países del sur de Europa comparten características en cuanto a la estructura dualista (Halleröd et al., 2015; Visser, 2009). En España se caracteriza por tener un mercado de trabajo rígido con una protección con diferencias entre quienes tienen un contrato indefinido -alta protección social, despido costoso y limitado- y los que tienen uno temporal —menor protección social y despido más fácil y menos costoso- (García-Espejo y Gutiérrez, 2011). Por lo tanto, se genera una provisión de bienestar dualizada y desequilibrada: muchos derechos para los que están en el centro del mercado de trabajo y pocos para los de la periferia (Rodríguez-Cabrero, 2011) Esta dualización del mercado laboral promueve una alta tasa de temporalidad, una alta rotación laboral y como consecuencia, el aumento del empleo precario o de baja calidad, incrementando el riesgo de pobreza que sufren los trabajadores (Tejero, 2017), en este sentido, Amuedo-Dorantes y Serrano-Padial (2010), señalan que tener un contrato temporal no sólo aumenta la probabilidad de pobreza actual sino también de pobreza futura mediante un efecto indirecto que aumenta las posibilidades de tener en el futuro un tipo de contrato con un mayor riesgo de pobreza.

\section{Pobreza dinámica}

Partimos del concepto de pobreza persistente que Eurostat introduce para tratar de analizar la situación de las personas que se encuentran por debajo del umbral de la pobreza, y que también lo han estado durante al menos dos de los tres años anteriores. 
Para ello se crea el indicador de tasa de riesgo de pobreza persistente, que capta a las personas que son particularmente vulnerables al riesgo persistente de pobreza durante períodos de tiempo realmente largos. La investigación sobre la pobreza dinámica se basa en la idea de la importancia que tiene el análisis del riesgo de pobreza laboral en los individuos, desde una perspectiva longitudinal.

El estudio de los aspectos dinámicos de la pobreza conduce a una mejor comprensión de la naturaleza de la pobreza y del tipo de individuos que la sufren. Conocer qué es lo que determina las entradas y salidas a la pobreza mejorará la explicación del concepto en sí mismo. Diversas investigaciones sobre pobreza dinámica se han centrado en el estudio de su cronicidad, diferenciando distintos tipos de pobreza en función de su duración y severidad, entre la pobreza crónica y la transitoria. Cantó et al. (2008, 2009 y 2012) resaltan la importancia, no solo de distinguir entre aquellos que persisten un año tras otro en la pobreza de los que caen en la misma de forma transitoria, sino que se acercan a la distinción propuesta por Bane y Ellwood (1986) para dividir a los pobres transitorios en recurrentes y no recurrentes. Estos dos subgrupos se diferencian en que, mientras los primeros viven diversos periodos de pobreza intercalados con periodos de no pobreza, los últimos solo se sitúan en ella ocasionalmente. Para determinar la cronicidad o la transitoriedad de la pobreza, habría que medir periodo a periodo y observar qué hogares y qué personas caen en ella, teniendo en cuenta el número de períodos y la duración total de esos episodios (Tejero, 2018). En este sentido, de la literatura revisada se desprenden diferentes hallazgos importantes en relación a las posibilidades de recurrencia, así Ayllón (2013) señala que el hecho de experimentar la pobreza en un periodo determinado, aumenta po sí mismo las posibilidades de volver a sufrir la pobreza en el futuro, igualmente sugiere que hay ciertas características observadas y no observadas que persisten en el tiempo y que podrían hacer que las personas sean sucesivamente pobres. Desde ese mismo enfoque, propone que el diseño de políticas debería centrarse en las transferencias de ingresos, si la persistencia de la pobreza se debe principalmente a las experiencias de pobreza del pasado, al objeto de ayudar a las personas a superar el umbral de la pobreza y así romper la espiral de pobreza en el futuro o, en el caso de pobreza transitoria, para hacer frente a las crisis temporales. Por el contrario, si la pobreza es crónica, las políticas más eficaces serían las orientadas a la protección de la adversidad, por ejemplo, la empleabilidad a través de la educación o la formación, mejorar la conciliación de la vida laboral y familiar, apoyar a las familias con niños y adolescentes, y políticas de integración de los inmigrantes.

\section{a. Pobreza temporal, recurrente y permanente}

Tejero (2018), señala que la perspectiva longitudinal permite ir más allá de la concepción tradicional y dicotomizada de la pobreza que reconoce solo dos situaciones, pobre o no pobre. Plantea que se necesita una tipología que tenga en cuenta la influencia de tiempo y define tres tipos de pobreza dinámica; pobreza permanente, pobreza recurrente y pobreza temporal, la primera está relacionada con la que Cantó et al (2012) definía como pobreza crónica, las dos siguientes están encuadradas con la pobreza transitoria recurrente y pobreza transitoria no recurrente. Para ello sugiere que la pobreza permanente se refleja en aquellas familias y trabajadores que persisten varios años en la pobreza y que se encuentran en una situación social y económica de mayor exclusión social. Se caracteriza porque las personas se mantienen por debajo de la tasa de pobreza durante un período prolongado de tiempo. Cuanto más tiempo se transcurre en este tipo de pobreza, más disminuirán sus recursos acumulados y su capacidad de ahorro (Cantó, 2002), por lo que se exponen a un continuo descenso de calidad de vida (Whelan et al. 2004) Por otra parte, señala que la pobreza recurrente es aquella que sufren las personas debido a la movilidad de sus ingresos provocando entradas y salidas de la pobreza en varias ocasiones. Esta es la que padecen aquellas personas que viven episodios alternativos, trabajador pobre/trabajador no pobre. Cantó (2002) apunta que el hecho de salir de la pobreza no es suficiente si no va seguido de un período suficientemente largo fuera de ella. Arranz y Canto (2008), sugieren que estas entradas y salidas a la pobreza se deben a la movilidad de los ingresos que suelen ser de corto alcance, de manera que los ingresos solo sirven para superar la línea de la pobreza, no siendo suficientes para mejorar su bienestar. Por último, se refiere a la pobreza temporal cuando las familias sufren etapas aisladas de pobreza. Estas las experimentan durante un período corto de tiempo. Según Arranz y Garcia-Serrano (2009), esta es la pobreza más extendida, pero, a su vez, la menos grave, ya que consiguen escapar de forma natural de la pobreza (Smith y Middleton, 2007). Esta situación pasajera, no tiene porqué condicionar sus oportunidades laborales, ni descender su nivel de bienestar.

En cuanto a los colectivos y los motivos que llevan a la pobreza de las distintas tipoogías, en la mayoría de los casos la pobreza permanente está ligada a la falta de trabajadores en el hogar y a los hogares monoparentales con niños (OCDE 2001-2008), la pobreza recurrente en España está muy relacionada con la situación de los miembros del hogar en el mercado de trabajo, desempleo y temporalidad (Ayala-Cañon y Sastre, 2007 OCDE 2001) y, por último, la pobreza temporal está relacionada con espacios temporales entre dos trabajos o un período de baja renta (Atkinson y Hills, 2008). Este tipo de pobreza afecta principalmente a hogares donde hay un alto número de adultos y cuyos miembros tienen un nivel educativo relativamente alto (Tejero, 2018)

\section{b. Pobreza dinámica en España}

Según Eurostat (2018), para el año 2016 en la UE-28, la tasa de pobreza persistente media para hogares unipersonales sin hijos era del 17,4\%. España estaba muy por 
debajo con un $8 \%$. Sin embargo, cuando se trataba del mismo tipo de familia, pero con hijos dependientes, España se encontraba por encima de la media $(21,9 \%)$ con un $24,5 \%$ Este mismo patrón se repitió en los hogares compuestos por dos o más adultos sin hijos, cuya tasa de pobreza persistente media era del 6,6\% y España (10\%) junto con Serbia $(11,4 \%)$ y Croacia $(16,1 \%)$ registrando las tasas más elevadas. En el caso del mismo tipo de hogar, pero con hijos dependientes, la media estaba situada en el 11,9\% y España tenía una de las tasas más altas con un $19 \%$, junto con Rumanía $(27,8 \%)$. Concluyendo, en el caso de los hogares monoparentales con hijos dependientes y en los hogares con dos o más adultos con o sin hijos dependientes, España se sitúa siempre por encima de la media de la UE-28. Solo se sitúa por debajo de la media cuando se trata de hogares unipersonales sin hijos.

Profundizando más en el análisis de la pobreza en España, (Cantó et al. 2012) señala que el 44\% de la población española sufrió al menos un año de pobreza entre 1994 y 2000. Cuando se trata de pobreza crónica, con un período de siete años, España registra una tasa del $2,7 \%$, muy similar a Francia $(2,8 \%)$ y Reino Unido $(2,8 \%)$ y por debajo de países como Dinamarca $(1,0 \%)$ o Alemania $(1,9 \%)$. Con respecto a la perspectiva de pobreza transitoria recurrente (dos o más episodios en el período de seis años) la tasa de pobreza fue del $18,2 \%$, muy por encima de países como Portugal (13,8\%) o Reino Unido (10,5\%). En cuanto a la pobreza transitoria no recurrente (un episodio en el período de seis años) la tasa fue del $23,0 \%$, situándose por debajo de Portugal $(24,6 \%)$ y apenas por encima del Reino Unido (22,7\%). Desde una perspectiva internacional, en líneas generales España no sale mal parada, estaría situada en una posición intermedia, debido a que, aun teniendo una tasa de pobreza alta, la tasa de transitoriedad registrada es elevada.

España, a pesar de haber vivido un ciclo de expansión económica y, como consecuencia, crecimiento del empleo, no ha sido capaz de reducir la pobreza ni la pobreza laboral. Esto se debe, principalmente, a la baja capacidad del sistema de protección social español para corregir las desigualdades a través de transferencias monetarias, propiciado por la escasez de las mismas para las familias en general y, sobre todo, para los hogares con niños y/o con miembros empleados, teniendo un impacto limitado en la reducción de la pobreza (Laparra y Ayala 2009; Rodríguez- Cabrero, 2010 y 2011; Ayala et al, 2013; Tejero, 2018). Por otra parte, desde la perspectiva de la incidencia de los salarios en la capacidad de influir en la tasa de pobreza, Banyuls y Recio (2017), señalan que la pobreza laboral no tendría la explicación tanto en la falta de crecimiento económico como en la existencia de desequilibrios estructurales.

\section{c. Resultados sobre la pobreza dinámica en España}

De los resultados de algunos de los trabajos que han analizado la pobreza desde una perspectiva dinámica, bien con la diferenciación tipológica entre pobreza transitoria no recurrente, pobreza transitoria recurrente o pobreza crónica (Canto et al, 2012) o desde la diferenciación análoga realizada por Tejero $(2017,2018)$, en la que diferencia la pobreza temporal, la pobreza recurrente y la pobreza permanente, señalando a su vez, que los factores que son importantes desde una perspectiva estática (relativos al hogar y a la participación laboral de sus miembros) son también importantes a la hora de analizar la cronicidad de la pobreza laboral, aunque deduce que la influencia de estos factores es diferente en función del tipo de pobreza que se esté padeciendo. De sus resultados se evidencian las siguientes cuestiones; en primer lugar, emergen dos niveles en cuanto al tipo de trabajo, es decir, trabajo por cuenta ajena y trabajo por cuenta propia. En ambos casos se analizan como afectan los siguientes factores; aspectos demográficos tales como la edad o el género, por otra parte, la intensidad laboral, hijos dependientes, tipo de contrato tipo de jornada o nivel de estudios principalmente.

En cuanto a la perspectiva de los factores que repercuten a la pobreza de los trabajadores por cuenta ajena, la pobreza laboral temporal está más condicionada por aspectos relativos a aspectos demográficos o a la situación concreta del individuo como por ejemplo, el género, el estado civil o el tipo de contrato y, en relación al hogar, tiene influencia la presencia de dependientes y la recepción de transferencias, por tanto, tienen más probabilidad de experimentar pobreza temporal, desde el punto de vista de género las mujeres, las personas con contratos temporales, así como aquellos hogares individuales o de dos adultos sin niños dependientes. En cambio, la pobreza recurrente está condicionada por la presencia de dependientes en el hogar, pero cobran mayor importancia los factores relativos al capital humano como son el nivel de estudios alcanzado. En este sentido tendrán más probabilidad de experimentar pobreza recurrente las personas que pertenecen a hogares con mayor presencia de menores dependientes, una intensidad laboral baja, bajos salarios ${ }^{4}$ y que no hayan acabado estudios superiores terciarios. Por último, en la pobreza persistente vuelve a ser importante determinados aspectos demográficos, así como también el capital humano, pero cobra especial importancia la composición del hogar, en mayor medida que en la pobreza temporal o recurrente. Por lo tanto, tienen más posibilidades de caer o permanecer en la pobreza persistente, en relación al género los hombres y en cuanto a la edad los jóvenes, asimismo, aquellas personas que tienen jornada de trabajo a tiempo parcial, bajos salarios, hogares con necesidades altas (con mayor presencia de menores dependientes), bajos recursos (intensidad laboral baja) y menor nivel de estudios.

Si se analiza la situación de la pobreza dinámica desde la perspectiva de los trabajadores por cuenta propia, se evidencia que los factores relacionados determinados aspectos de-

4. La presencia de menores dependientes, la intensidad laboral y los salarios bajos, son factores que inciden tanto en la pobreza recurrente como en la persistente, siempre dependiendo de cómo afecten el resto de factores.

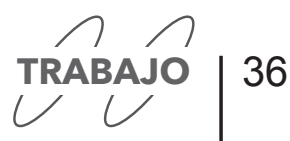


mográficos como, por ejemplo, el estado civil —concretamente aquellas personas que están casadas - y la baja intensidad laboral del hogar tienen influencias altas y son determinantes en la pobreza laboral temporal y recurrente, por último, el perfil de persistencia de los trabajadores por cuenta propia está más influenciado por la carencia de ingresos por inactividad.

Por último, los resultados de estos estudios sugieren que, desde una perspectiva longitudinal, los trabajadores por cuenta propia tienen un riesgo de pobreza laboral mucho más alto que los trabajadores por cuenta ajena. Por tanto, el concepto de trabajador pobre en el caso español se proyecta hacia la concepción de que los trabajadores por cuenta propia tienen un riesgo mucho mayor en cualquiera de las situaciones de pobreza dinámica (Tejero, 2018).

\section{Conclusiones y propuestas}

El presente trabajo ha pretendido, a través de una revisión de la literatura, contextualizar la situación de la pobreza y exclusión social en España, para luego profundizar en la pobreza laboral y el desempleo. Partimos de que uno de los objetivos principales recogidos en la Estrategia de Europa 2020 era reducir, en el período 2009-2019, 20 millones de personas de las registradas en riesgo de pobreza y de exclusión social. Para el caso español, el compromiso fue rebajar en el mismo período, entre 1.400 .000 y 1.500 .000 personas de la mencionada situación. La realidad es que a falta de un año de que se cumpliera e plazo, y a pesar de la mejora en los cuatro últimos años, no solo no se ha cumplido el compromiso, sino que está por encima de la tasa de la situación de partida. Para que España cumpla el objetivo habría que reducir en términos absolutos en 2,6 millones de personas la tasa de pobreza y exclusión social, que en 2018 se situaba en un $26,1 \%$ (12.188.288 personas) de la población española. La tasa española es la séptima más alta de los países miembros de la UE y se sitúa con un $4,2 \%$ por encima de la media. En cuanto al perfil de las personas que forman parte de esta tasa, las mujeres registran un riesgo más elevado de pobreza y exclusión social que los hombres, los jóvenes más que los mayores, y significativamente es relevante, que los trabajadores, proporcionalmente, tienen mayor riesgo que los desempleados. Con respecto a los hogares, los monoparentales son los que, con un $50 \%$, sufren más esta situación.

Por otra parte, el desempleo tiene un fuerte impacto sobre la pobreza, sobre todo por la proporción de hogares en los que todos los miembros activos están desempleados. Tras la crisis que irrumpió en 2007, el desempleo en España se eleva de un 8,6\% al 26,0\% en cinco años. La proporción de hogares en los que todos los miembros activos estaban desempleados aumentó de un $2,6 \%$ al $10,5 \%$. Las consecuencias no son visibles solo en el momento en que se viven, sino que tienen efectos intergeneracionales, afectando de forma significativa en el bienestar futuro de los hijos.
Con respecto a los trabajadores pobres - aquellas personas que, aun teniendo un empleo remunerado, su salario no les permite disponer de los recursos necesarios para satisfacer sus necesidades-, el peso de la repercusión de las características del hogar influye más en la probabilidad de riesgo de pobreza que la dimensión individual. Esto explica que no todos los trabajadores que tienen un salario bajo son pobres, ni todos los que tienen un salario alto están exentos de serlo, fundamentalmente, porque está ligado a la influencia de las características del hogar al que pertenecen y a las circunstancias sus miembros. En lo relativo a la perspectiva individual, los factores que inciden en el riesgo de pobreza están relacionados con la precariedad del mercado laboral; bajos salarios, alta tasa de temporalidad y alta rotación laboral, comprometiendo no solo la situación presente, sino que aumenta la posibilidad y el riesgo de pobreza futura provocada por la necesidad de optar a contratos más precarios y con un mayor riesgo de pobreza. La estructura dualista del mercado laboral español, con una protección diferente entre aquellos que tienen un contrato indefinido y los que tienen un contrato temporal, no hace otra cosa que generar una brecha entre los que están en el centro del mercado y los que están en la periferia, abocados, por la temporalidad y las consecuencias de largos períodos de desempleo a un mayor riesgo de pobreza y exclusión social.

Por último, desde la perspectiva de la pobreza dinámica, cuando se trata de trabajadores por cuenta ajena, la pobreza laboral temporal o transitoria no recurrente incide más, desde el punto de vista de género; en las mujeres, con respecto al tipo de contrato; en los contratos temporales y, con respecto al tipo de hogar; en aquellos hogares individuales o de dos adultos, pero sin niños dependientes. Con respecto a la pobreza transitoria recurrente, está relacionada con mayor presencia de menores dependientes, intensidad laboral baja, bajos salarios y la carencia de estudios secundarios. Finalmente, cuando se trata de pobreza persistente o crónica, aquí en el caso de género, los hombres son los más desfavorecidos y, en cuanto a la edad; los más desfavorecidos son los jóvenes y aquellas personas que tienen jornada de trabajo a tiempo parcial, bajos salarios, hogares con mayor presencia de menores, baja intensidad laboral y menor nivel de estudios. Sin embargo, cuando se trata de trabajadores por cuenta propia, con respecto a la pobreza laboral transitoria no recurrente (temporal) y también la pobreza laboral recurrente, los factores más determinantes son, el estado civil - estar casados_y la baja intensidad laboral del hogar. En cuanto a la pobreza laboral persistente, el principal causante es la carencia de ingresos por inactividad. Desde la perspectiva de pobreza dinámica, los trabajadores por cuenta propia tienen un mayor riesgo de pobreza laboral que los trabajadores por cuenta ajena.

\section{Propuestas}

Las propuestas para tratar de reducir la pobreza y exclusión social, así como la pobreza laboral, deberán ir encaminadas al diseño de políticas que tengan la ambición y el 
compromiso de corregir la deriva a la que están sometidas las personas que sufren esta situación. Estas políticas deberían sustanciarse en los motivos que inciden para que los colectivos afectados ingresen en la pobreza y en las barreras que hacen que su salida sea un reto prácticamente imposible. De esta manera, sería interesante el aumento de las transferencias sociales, teniendo en cuenta la importante repercusión que tienen estas aportaciones para hacer frente a la pobreza monetaria. A su vez las transferencias sociales, además de ayudar a las personas a superar el umbral de la pobreza y romper la espiral de pobreza en el futuro, para el caso de las personas con riesgo de pobreza transitoria, son eficaces para hacer frente a las crisis temporales. Para el caso de las personas que están en una situación de pobreza crónica, las políticas más eficaces serían las orientadas a la empleabilidad a través de la educación o la formación, mejorar la conciliación de la vida laboral y familiar, apoyar a las familias con niños y adolescentes, y las políticas de integración de los inmigrantes. 


\section{Referencias bibliográficas}

Airio, I. 2008. Change of a norm? In-work poverty in a comparative perspective. Helsinki: The Social Insurance Institution of Finland, Research Department

Albort-Morant, G., \& Ribeiro-Soriano, D. (2016). A bibliometric analysis of internationalimpact of business incubators. Journal of Business Research, 69(5), 1775-1779.

Andress, H., \& Lohmann, H. (2008). The working poor in europe : employment, poverty and globalization . Northampton, MA: Edward Elgar.

Aragón, J., Cruces, L.F., Martínez, A., \& Otaegui, A. (2012). Trabajadores pobres y empobrecimiento en España. Zerbitzuan (52), 1-10. http://dx.doi.org/10.5569/1134-7147.52.07

Ayala, L., Cantó, O., \& Rodríguez, J. (2017). Poverty and the business cycle: A regional panel data analysis for Spain using alternative measures of unemployment. The Journal of Economic Inequality, 15(1), 47-73. https://doi.org/10.1007/s10888-016-9343-5

Ayllón, S. (2013). Understanding poverty persistence in Spain. SERIEs : Journal of the Spanish Economic Association, (2), 201-233. Retrieved from https://dialnet.unirioja.es/servlet/ oaiart?codigo $=5502478$

Bardone, L. y A-C. Guio. 2005. "In-work poverty. New commonly agreed indicators at EU level". Statistics in focus, 5/2005.

Cantó, Olga, \& Gradín, Carlos, \& Del Río, Coral (2012). Pobreza crónica, transitoria y recurrente en España. Revista de Economía Aplicada, XX(58), 69-94, https://www.redalyc. org/articulo.oa?id=969/96924442003

García, Carlos y Arranz, José M., 2014, "Evolución de la desigualdad salarial en los países desarrollados y en España en los últimos treinta años", en FOESSA, VII informe sobre exclusión y desarrollo social en España. FOESSA, Madrid.

García, Isabel e lbáñez, Marta, 2007, "Los trabajadores pobres y los bajos salarios en España: un análisis de los factores familiares y laborales asociados a las distintas situaciones de pobreza”, en Empiria. Revista de metodología de ciencias sociales, núm.14, pp. 41-67

García-Espejo, Isabel and Gutiérrez, Rodolfo (2011). "Growing Employment and Persisting Inequalities". In: Fraser, N.; Gutiérrez, R. and Peñas- Casas, R. Working Poverty in Europe. Hampshire: Palgrave-McMillan.

Gregg, P., Scutella, R., Wadsworth, J. (2010). Reconciling workless measures at the individual and household level. J. Popul. Econ. 23, 139-167
Halleröd, Björn; Ekbrand, Hans and Begstsson, Mattias (2015). “In-Work Poverty and Labour Market Trajectories: Poverty Risks among the Working Population in 22 European Countries". Journal of European Social Policy, 25(5): 473-488.

Llano, J.C. (2019). El Estado de la pobreza. Seguimiento del indicador de pobreza y exclusión social en España 2008-2018. EAPN-ES

Martínez-Martín, R., García-Moreno, J., \& Lozano-Martín, A. (2018). Trabajadores pobres en España. El contexto de la crisis económica como marco para comprender la desigualdad Papeles de Población, 24(98), 185-218. https://doi.org/10.22185/24487147.2018.98.40

Mata, Adriana, 1999, Definiciones internacionales y futuro de las estadísticas del subempleo, Oficina Internacional del Trabajo, Ginebra.

OIT, 2015, Informe Mundial sobre Salarios 2014/2015. Salarios y desigualdad de ingresos. Organización Internacional del Trabajo, Ginebra.

Peñas-Casas, R. y M. Latta. 2004. Working poor in the European Union. Luxemburgo: European Foundation for the Improvement of Living and Working Conditions.

Ponthieux, S. 2010. "Assessing and analysing in-work poverty risk". Pp. 307-328 en Income and living conditions in Europe, editado por A. B. Atkinson y E. Marlier. Luxemburgo: Eurostat Statistical Books.

Rodríguez-Cabrero, Gregorio (2011). "The Consolidation of the Spanish Welfare State (19752010)". In: Guillén, A. M. and León, M. (eds.). The Spanish Welfare State in European Context. Farnham: Ashgate.

Strengmann-Kuhn, W. 2002. "Working poor in Europe: a partial basic income for workers?" Paper presentado en el 9th International Congress of Basic Income European Network, 12-14 septiembre, Ginebra. https://doi.org/10.2139/ssrn.386540

Tejero, A, 2017. Permanencia en la pobreza laboral: la influencia de la pobreza pasada en la presente. Revista Española de Investigaciones Sociológicas, 157: 141-162. http://dx.doi org/10.5477/cis/reis.157.141

Tejero, A., 2018. Pobreza laboral en España. Un análisis dinámico. Revista Internacional de Sociología 76(2): e096. https://doi. org/10.3989/ris.2018.76.2.16.54

Visser, Jelle; Beentjes, Marieke; Van Gerven, Minna and Di Stasio, Valentina (2009). "The Quality of Industrial Relations and the Lisbon Strategy”. In: European Commission (ed.). Industrial Relations in Europe 2008. Bruselas: European Commission. 